\title{
Efficacy of a Combination of Simvastatin and Poly(DL-lactic-co-glycolic acid) in Stimulating the Regeneration of Bone Defects
}

\author{
Kyl Assaf, Eliana Aparecida de Rezende Duek, Newton Maciel Oliveira* \\ Pontifical Catholic University of São Paulo - PUC-SP, \\ Praça Dr. José Ermírio de Moraes, 290, CEP 18030-095, Sorocaba, SP, Brazil
}

Received: March 29, 2012; September 9, 2012

\begin{abstract}
The proper healing of bone defects requires a bone graft or bone substitute and synthetic materials have been developed as alternatives to autografts and allografts. Poly(DL-lactic-co-glycolic acid) (PLGA) is a synthetic polymer widely used for bone healing because of its biocompatibility and biodegradability. PLGA scaffolds have also been used in drug delivery devices, such as in combination with simvastatin, to stimulate bone growth. In this work, we examined the usefulness of a combination of PLGA with simvastatin for treating bone defects. For this, two defects were created in rat calvaria and in half of the animals the right sides were filled with PLGA scaffolds while the other half received PLGA plus simvastatin; the left sides remained empty. The rats were killed for histological analysis after four and eight weeks. There was a significant increase in the amount of bone formation in the treated lesions, particularly those that received PLGA plus simvastatin.
\end{abstract}

Keywords: bone regeneration, PLGA, simvastatin, tissue engineering

\section{Introduction}

Under normal conditions, bone tissue heals well with conventional therapy through its capacity for spontaneous regeneration. However, for large defects and congenital bone deformities, a bone graft or bone substitute may be needed to improve healing ${ }^{1}$. Autografts require additional surgery at the donor site that can result in complications such as inflammation, infection and chronic pain ${ }^{2}$. Furthermore, the total amount of bone that can be harvested for autografts is limited and severely restricts the supply of material. On the other hand, allografts, which involve tissue from a donor, carry the risk of disease transmission ${ }^{2}$. These problems have led to the development of synthetic materials as alternatives to autografts and allografts for bone repair ${ }^{2}$.

The ideal synthetic bone graft should be osteoconductive, osteoinductive, biocompatible, bioresorbable, structurally similar to bone, easy to use and cost effective ${ }^{3}$. Poly(DL-lactic-co-glycolic acid) (PLGA) is a synthetic polymer with most of these features since it is biocompatible, non-toxic, biodegradable and the products of hydrolysis are easily metabolized by the organism ${ }^{4}$. Indeed, when used in surgery, PLGA scaffolds do not need to be removed ${ }^{4}$. PLGA scaffolds have been used in drug delivery devices ${ }^{5}$ to incorporate drugs such as simvastatin that promote osteoinduction.

Simvastatin belongs to the group of drugs known as statins that were introduced as cholesterol-reducing drugs but have since been used to increase bone mass ${ }^{2,6-8}$. The effects of statins on bone formation are associated with an increase in the expression of bone morphogenetic protein-2 (BMP-2) mRNA and enhanced vascular endothelial growth

*e-mail: newtonmo@hotmail.com factor (VEGF) expression, an important factor in osteoblast differentiation ${ }^{3,9}$. Statins can also reduce bone resorption by inhibiting the fusion of osteoclast precursors or by disrupting the actin ring of osteoclasts, leading to a decrease in the number of active osteoclasts ${ }^{10,11}$.

The bioavailability of simvastatin in the general circulation is low $(<5 \%)$ after oral administration because of extensive first-pass metabolism in the liver; this pharmacokinetic characteristic leads to a lower concentration of the drug in other tissues ${ }^{11}$. Since the successfulness of simvastatin in promoting bone formation in vivo depends on a high local concentration there have been continuous efforts to find an appropriate delivery system to increase the circulating concentrations of this drug ${ }^{11}$.

The aim of this study was to evaluate the efficacy of a combination of PLGA with simvastatin in stimulating the regeneration of bone tissue following damage. For this, we assessed the size of defects remaining four and eight weeks after implantation of a PLGA scaffold (with and without simvastatin) and examined the biocompatibility of the polymer and tissue regeneration based on histological analysis.

\section{Experimental}

\subsection{Scaffold fabrication}

Scaffolds were prepared using PLGA 50/50 (PURAC) dissolved in methylene chloride (Merck) $(10 \%, \mathrm{~m} / \mathrm{v})$. After complete dissolution of the polymer, simvastatin (Merck) was added to a final concentration of $1 \%$. The solution was then poured into a Petri dish to allow solvent evaporation at room temperature for 24 hours. 


\subsection{Experimental design}

Thirty-two male Wistar rats (250-300 g) were used. The animals were housed in plastic cages at $22{ }^{\circ} \mathrm{C}$ on a 12 hours light/dark cycle and received standard rodent chow and water ad libitum. The animal use described here was approved by an institutional Committee for Ethics in Animal Use of University of Paraiba Valley (UNIVAP) (protocol $n^{\circ}$ A051/CEP/2009) and the experiments were done within the general guidelines of the Brazilian Society for Laboratory Animal Science (SBCAL) and the principles of laboratory animal care (NIH publication 42-75, 2011)

\subsection{Surgical procedure}

The rats were anesthetized with $10 \%$ ketamine (100 mg. $\left.\mathrm{kg}^{-1}\right)$ and $2 \%$ xylazine hydrochloride $\left(6 \mathrm{mg} \cdot \mathrm{kg}^{-1}\right)$, both given intramuscularly, in a 1:1 ratio. The dorsal part of the cranium was shaved and aseptically prepared for surgery. A 20-mm-long incision was made in the scalp along the sagittal suture and the musculature and the periosteum were reflected to expose the parietal bone (Figure 1a). In each rat, two critical-size defects, $5.25 \mathrm{~mm}$ in diameter, were created in the dorsal part of the parietal bone, as described by Bosch et al. ${ }^{12}$. The defects were prepared with a trephine bur driven at low speed by a dental drill and cooled with copious phosphate-buffered saline, with care taken so as not to injure the bone edges and the dura mater (Figure 1b,c).

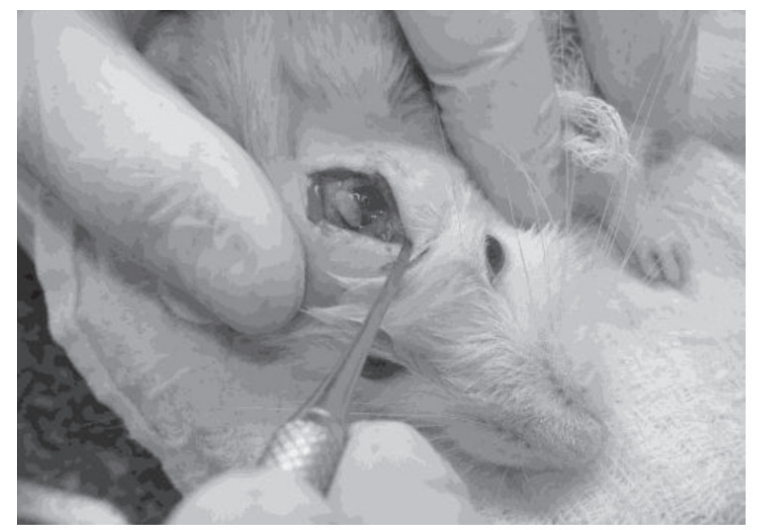

(a)

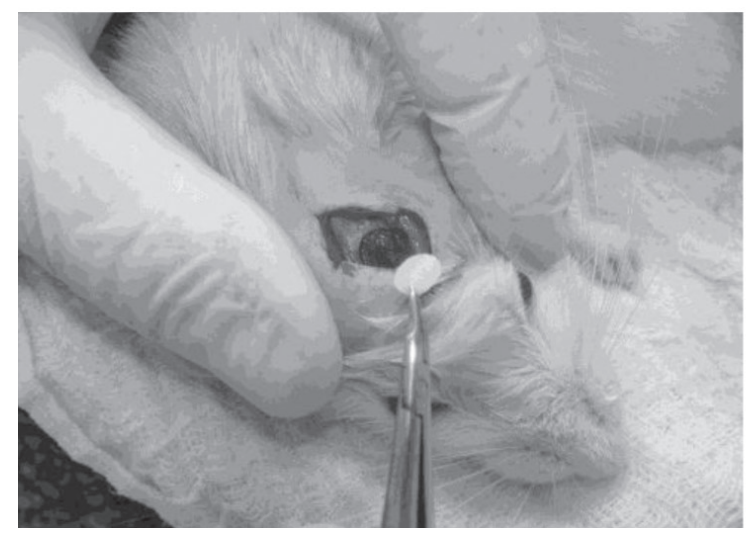

(c)
The rats were divided in two groups ( $\mathrm{n}=16$ each). The defect on the right side was always the experimental one and that on the left always the control that did not receive any treatment. In the first group, the defect on the right side was filled with PLGA alone while in the second group, simvastatin was added to the PLGA scaffold (Figure 1c). The scaffolds were sterilized by immersion in $70 \%$ ethanol for 1 hour and washed in saline prior to surgery. The periosteal incision was closed with 8-0 (Vicril ${ }^{\circledR}$, Ethicon) interrupted sutures (Figure 1d).

\subsection{Histological analysis}

Four and eight weeks after surgery, the rats were killed with an overdose of anesthetic and the parietal bones of each animal were carefully removed and fixed in Bouin solution for 8 hours, after which they were washed in water for 24 hours and decalcified in $4.13 \%$ EDTA solution for 30 days. Subsequently, the specimens were washed extensively in $0.1 \mathrm{M}$ sodium cacodylate buffer, $\mathrm{pH}$ 7.2, and segments containing defects were cut with a scalpel into two portions (experimental and control regions). All samples were dehydrated in a graded ethanol series, cleared in xylene and embedded in paraffin. Sections $\sim 3 \mu \mathrm{m}$ were stained with hematoxylin-eosin and examined with a light microscope. The area of the surgical lesion in each rat was expressed as the length of the remaining defect and was determined by image analysis using Nikon Imaging Software Elements Advanced Research Version 3.00.

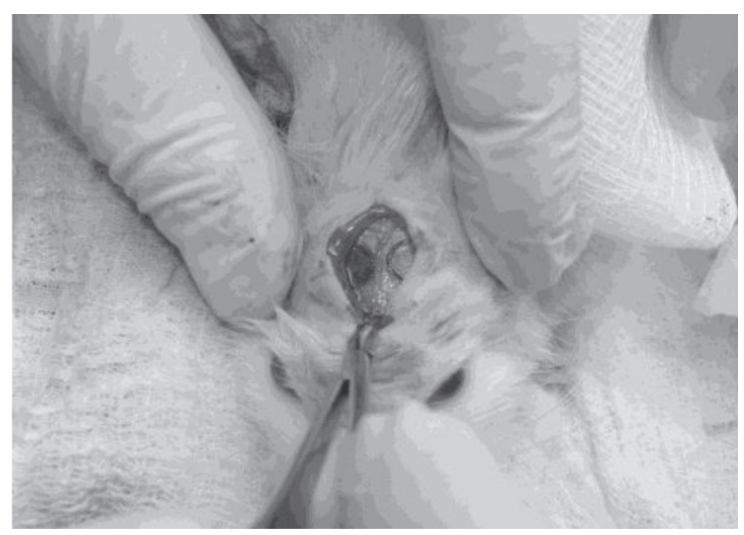

(b)

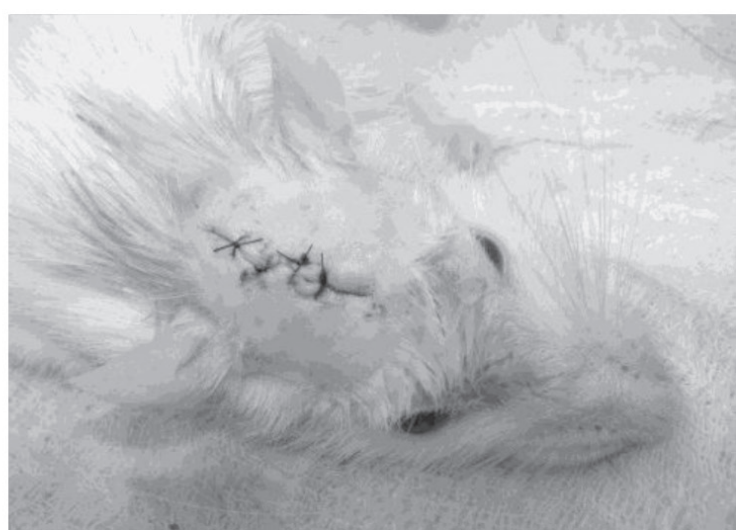

(d)

Figure 1. Surgical procedure. a) Bone tissue removal. b) Bilateral critical-size defects. c) Insertion of the polymer implant. d) Suture. 


\subsection{Statistical analysis}

The results, expressed as the mean \pm standard deviation (SD), were analyzed statistically by ANOVA followed by the Tukey post-hoc test. A value of $\mathrm{p}<0.05$ indicated significance.

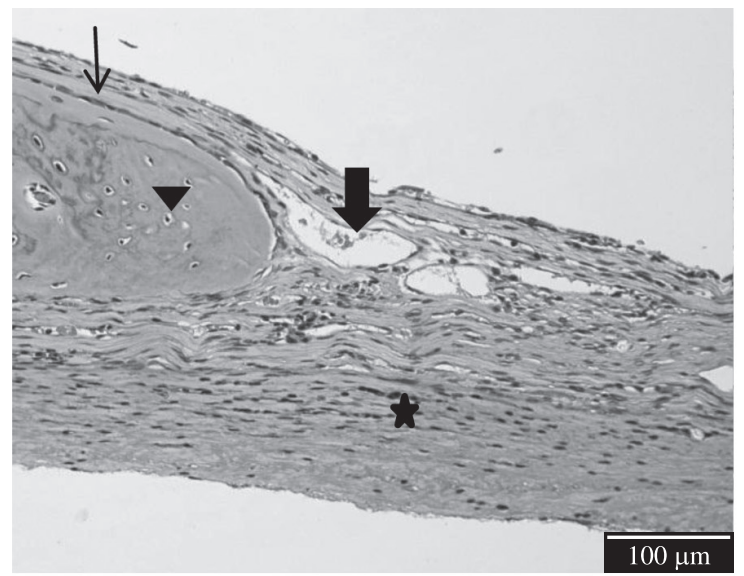

(a)

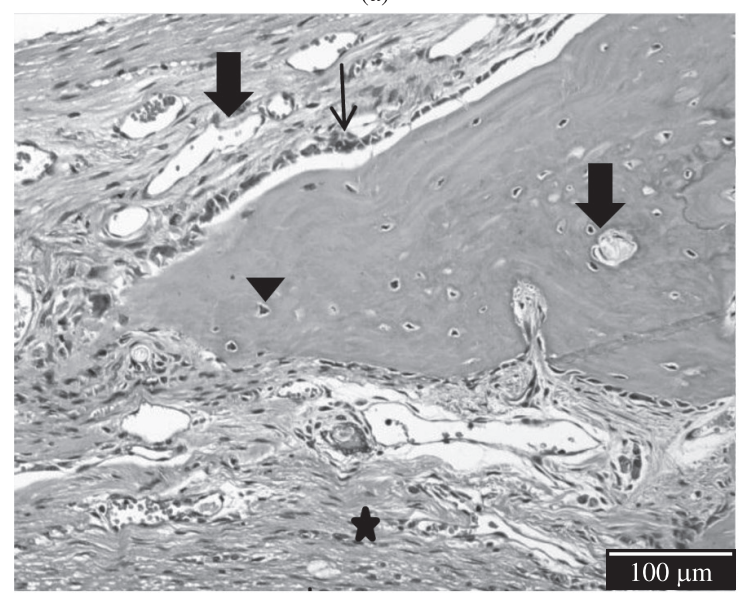

(c)

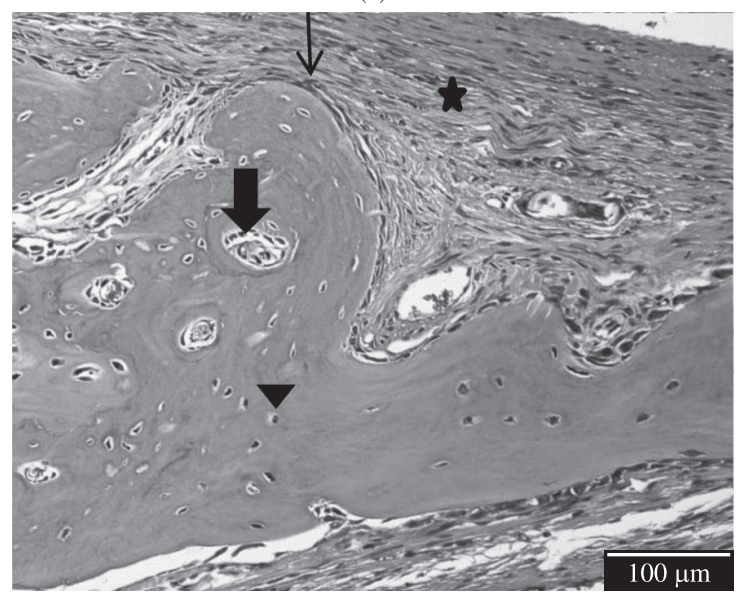

(e)

\section{Results}

\subsection{Histological analysis}

In the control group, after four weeks almost all of the defects were filled with a thin layer of loose connective tissue and some fibroblasts (Figure 2a). Bone formation, which

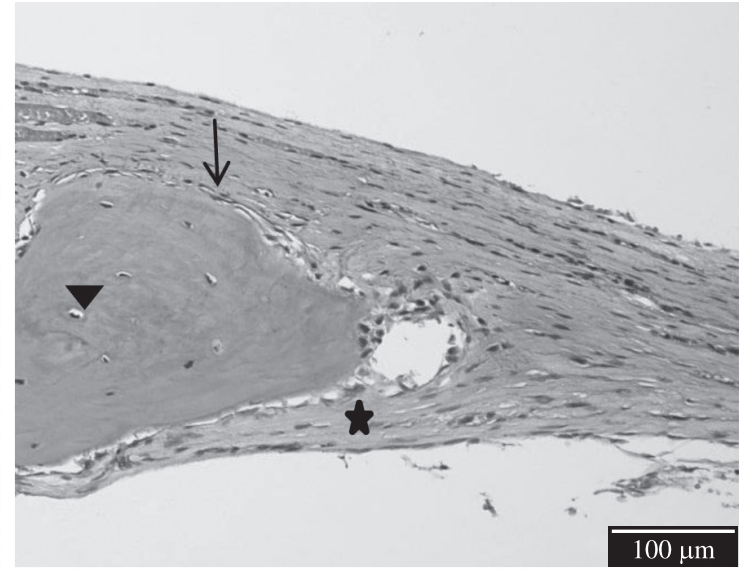

(b)

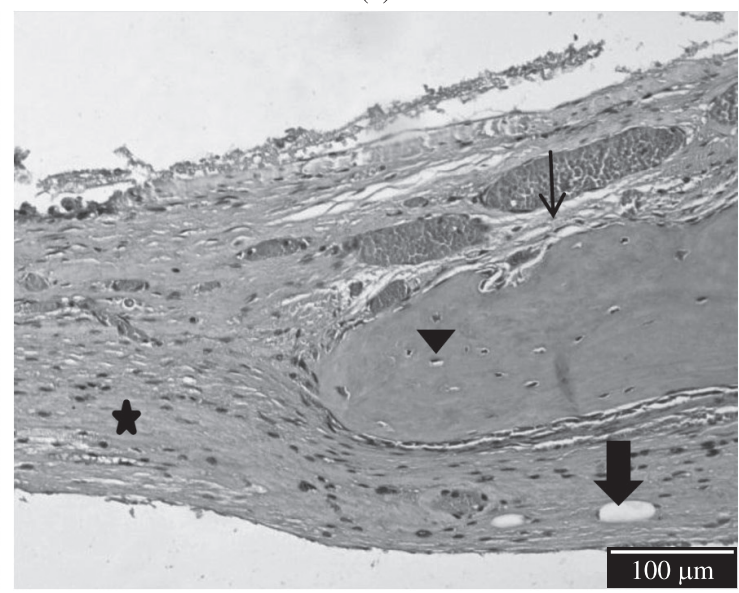

(d)

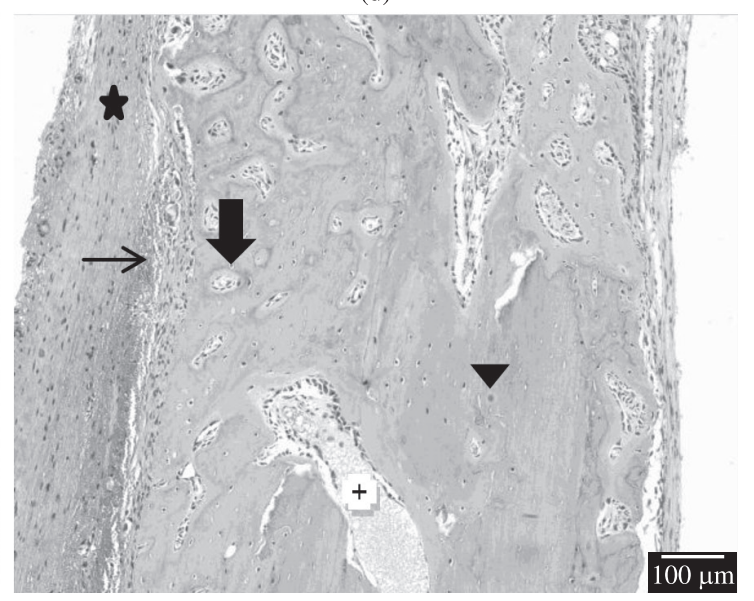

(f)

Figure 2. Histological appearance of defect edges. Note the presence of osteoblasts (thin arrows), osteocytes (arrowheads), blood vessels (thick arrows), polymer (cross) and connective tissue (stars). (a) and (b) Control group after 4 and 8 weeks, respectively. (c) and (d) PLGA group after 4 and 8 weeks, respectively. (e) and (f) PLGA and simvastatin group after 4 and 8 weeks, respectively. HE staining. 
progressed from the edge towards the center, was minimal. After eight weeks, the connective tissue contained thicker collagen fibers compared to the four-week period and the bone formation was greater. There was no defect closure in any period (Figure $2 b$ ).

In the PLGA group, the layer of connective tissue in the area of the lesion was thicker than in the control group after four weeks. The neotissue contained more blood vessels than control tissue, as well as polymer debris surrounded by macrophages, but no acute inflammatory response. There was greater bone formation at the edge of the defect than in the control group and the neotissue exhibited blood vessels, osteoblasts and some osteoclasts at the periphery (Figure 2c). After eight weeks, the same aspects were observed but bone formation was increased and bone tissue was also present within some defects. There was no defect closure in any period (Figure 2d).

In defects grafted with PLGA and simvastatin, there was bone formation within the defects and increased bone formation at the edges after four weeks compared to defects treated with PLGA alone. Blood vessels, osteoblasts and polymer debris surrounded by macrophages were also observed, as in the PLGA group, but there was no acute inflammatory response (Figure 2e). After eight weeks, the remaining surgical area was very small but no defect showed complete regeneration. Blood vessels and osteoblasts were observed in higher numbers compared to after four weeks and there were also some macrophages (Figure 2f).

\subsection{Defect size}

Measurement of the defect size after four weeks showed that bone formation, which resulted in smaller (shorter) defects, was greater in the experimental groups than in the control group, and greater in the PLGA plus simvastatin group than in defects treated with PLGA alone; similar findings were observed after eight weeks (Figure 3). Figure 4 shows the histological appearance of these tissues.

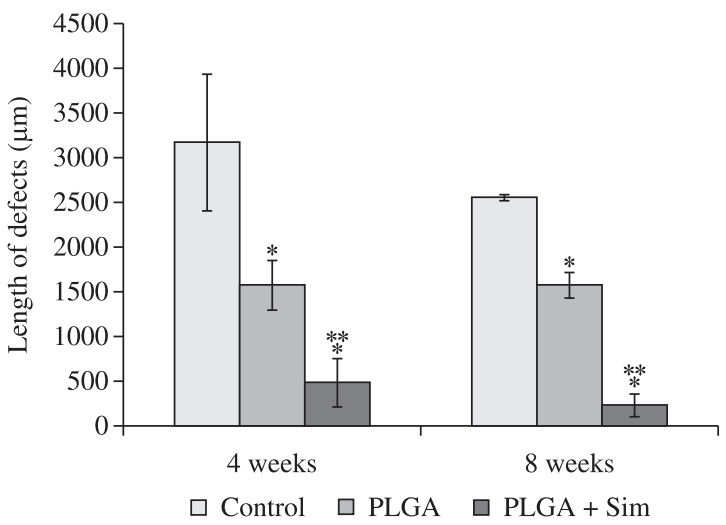

Figure 3. Distance between the edges of the defects in the control and experimental groups after four and eight weeks. A decrease in the distance between edges indicated greater healing of the lesion. The columns are the mean \pm SD of 16 rats in experimental groups and 32 in control group. ${ }^{*} \mathrm{p}<0.01$ compared to the control group, $* * \mathrm{p}<0.01$ compared to the PLGA group.

\section{Discussion}

In this study, we used a bilateral critical-size bone defect to analyze the usefulness of PLGA as a scaffold for bone regeneration and for delivering simvastatin, a drug with osteoinductive activity, in rat calvaria. None of the control (untreated) rats showed complete defect regeneration, even after eight weeks. This finding agreed with Bosch et al. ${ }^{12}$ who determined that defects $5 \mathrm{~mm}$ in diameter were a critical size for rat parietal bone. Empty defects contained essentially connective tissue, in agreement with other reports in which large bone defects remained unrepaired and contained some fibrous connective tissues in the damaged region $^{13}$.

In rats treated with PLGA or PLGA plus simvastatin, none of the defects regenerated completely, even after eight weeks. Ciéslik et al. ${ }^{14}$ showed that defects in rabbit mandible required 48 weeks for complete filling and healing and that the process was similar to the control group in which there was total disappearance of osteoblasts only after 24 weeks.

Rats treated with PLGA or PLGA plus simvastatin showed a marked increase in bone tissue after four weeks of repair when compared with the control group. This increase in bone tissue may be related to the presence of blood vessels, particularly in the PLGA plus simvastatin group. Some studies have shown that an increase in the number of new blood vessels around critical- and non-critical-size defects may indicate angiogenesis that precedes bone repair in defects of both sizes ${ }^{15}$

Although the polymer-treated groups showed polymer debris surrounded by macrophages there was no acute inflammatory response; this finding characterized the polymer as a biocompatible material because its degradation did not cause an important inflammatory or infective response. A study of the biological properties of PLGA-based composites grafted into rabbit mandible showed that PLGA enhanced cellular activity, with an increase in the number of numerous osteoblasts, indicating that this material was biocompatible ${ }^{14}$.

The enhanced bone formation seen in the PLGA group reflected the ability of the polymer to act as a scaffold for bone growth and this growth was increased further by the presence of simvastatin. Although PLGA stimulates bone formation in tooth sockets by providing a three-dimensional scaffold for bone deposition, greater bone formation is observed in response to the osteogenesis stimulated by simvastatin $^{11}$. In contrast, some studies have suggested that the ability of simvastatin to enhance bone formation is more related to its anti-absorptive activity than to its capacity to induce osteogenesis ${ }^{10,16,17}$.

The marked reduction in the size of the defect four and eight weeks after treatment with PLGA and PLGA plus simvastatin compared to the control group, as well as between the two treated groups, clearly showed that the presence of PLGA had a beneficial effect on wound healing and that this response was enhanced by simvastatin. 


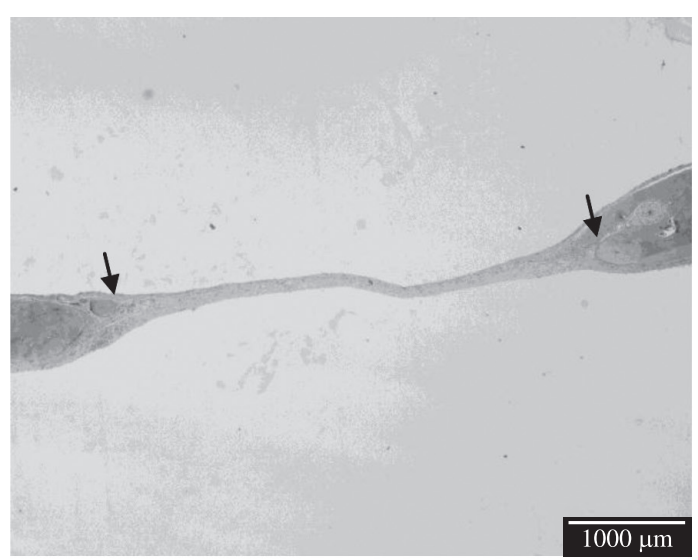

(a)

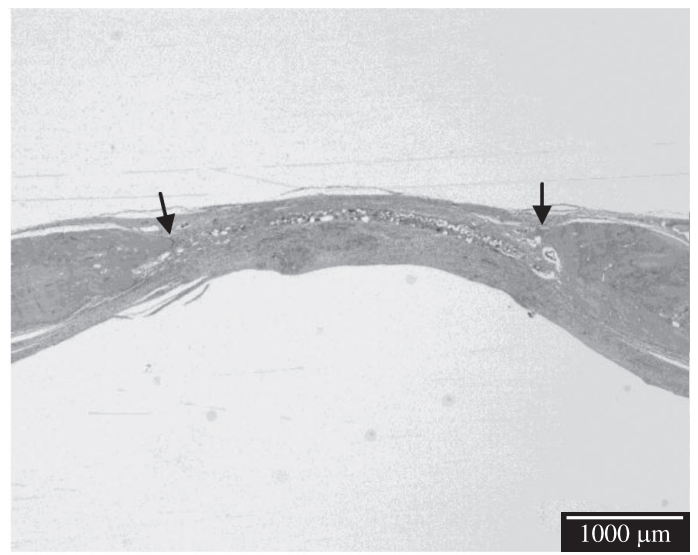

(c)

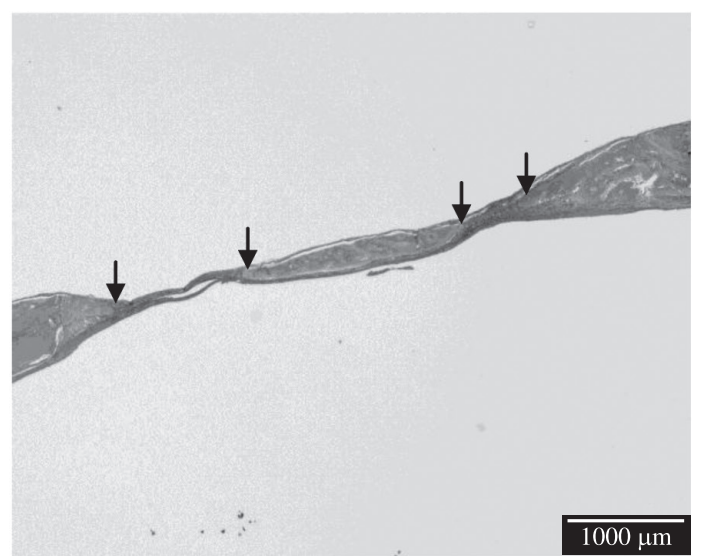

(e)

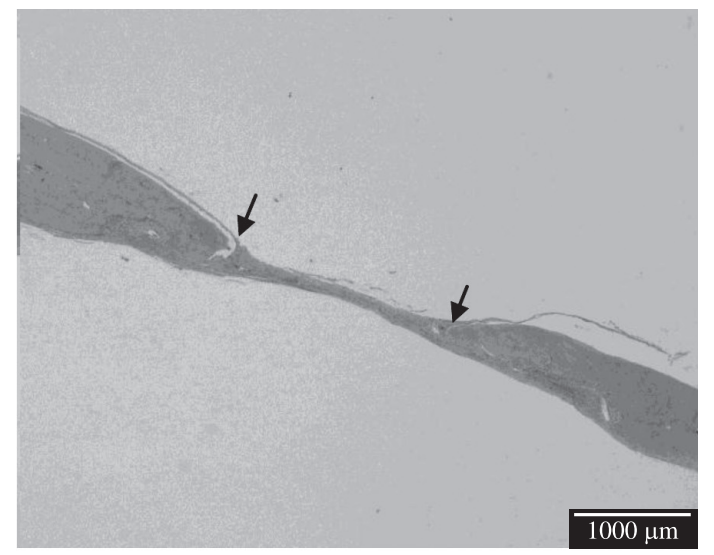

(b)

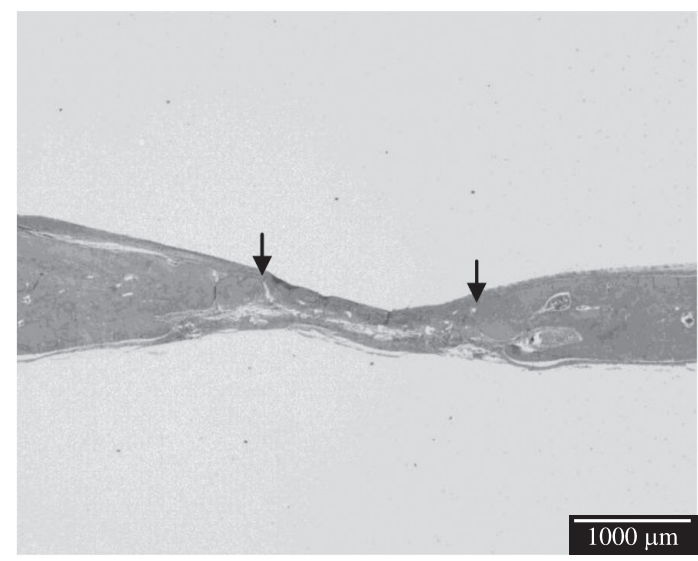

(d)

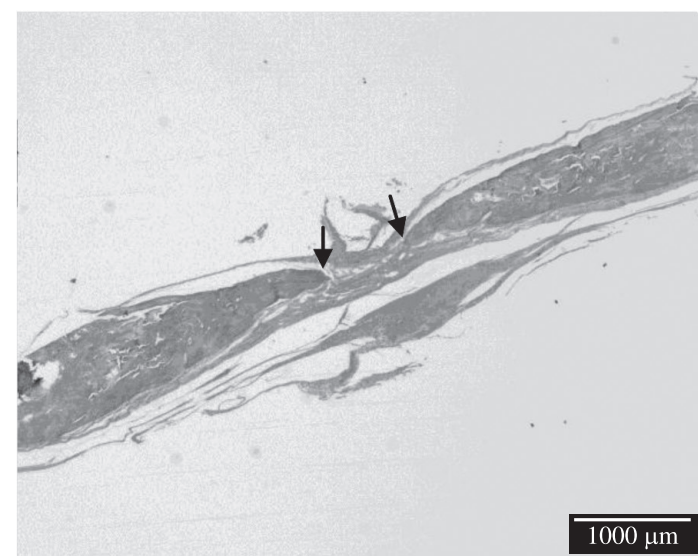

(f)

Figure 4. Histological analysis of the distance between defect edges. (a) and (b) Control group after 4 and 8 weeks, respectively. (c) and (d) PLGA group after 4 and 8 weeks, respectively. (e) and (f) PLGA and simvastatin group after 4 and 8 weeks, respectively. The arrows indicate the edges of the remaining defect. In the Figure $4 \mathrm{f}$, the presence of bone within the defect created two small remaining defects instead of one, as shown in other figures. HE staining.

\section{Conclusion}

Although none of the defects showed complete regeneration there was, nevertheless, an increase in bone formation in defects treated with PLGA, particularly those that received simvastatin concomitantly. Based on these findings, we conclude that the PLGA scaffold containing simvastatin was a useful osteoconductive and osteoinductive combination that improved the regeneration of critical-size bone defects.

\section{Acknowledgements}

The authors thank André Messias for help with the statistical analysis. This work was supported by the PUC-SP Teaching and Research Commission (CEPE). 


\section{References}

1. Puppi D, Chiellini F, Piras AM and Chiellini E. Polymeric materials for bone and cartilage repair. Progress in Polymer Science. 2010; 35:403-440. http://dx.doi.org/10.1016/j. progpolymsci.2010.01.006

2. Jeon JH, Thomas MV and Puleo DA. Bioerodible devices for intermittent release of simvastatin acid. International Journal of Pharmaceutics. 2007; 340:6-12. http://dx.doi.org/10.1016/j. ijpharm.2007.03.007

3. Giannoudis PV, Dinopoulos $\mathrm{H}$ and Tsiridis E. Bone substitutes: An update. Injury. 2005; 36: 20-27. http://dx.doi.org/10.1016/j. injury.2005.07.029

4. Mano JF, Sousa RA, BoeselLF, Neves NM and Reis RL. Bioinert, biodegradable and injectable polymeric matrix compositesfor hard tissue replacement: state of the art and recent developments. Composites Science and Technology. 2004; 64:789-817. http://dx.doi.org/10.1016/j. compscitech.2003.09.001

5. RashidiH, Ellis MJ, Cartmell SH and Chaudhuri JB. Simvastatin release from poly(lactide-co-glycolide)membrane scaffolds. Polymers. 2010; 2:709-718. http://dx.doi.org/10.3390/ polym 2040709

6. Stein D, Lee Y, Schmid MJ, Killpack B, Genrich $\mathrm{MA}$, Narayana $\mathrm{N}$ et al. Local simvastatin effects on mandibular bone growth andinflammation. Journal of Periodontology. 2005; 76:1861-1870. http://dx.doi. org/10.1902/jop.2005.76.11.1861

7. Lee Y, Schmid MJ, Marx DB, Beatty MW, Cullen DM, Collins ME et al. The effect of local simvastatin delivery strategieson mandibular bone formation in vivo. Biomaterials. 2008; 29:1940-1949. http://dx.doi.org/10.1016/j. biomaterials.2007.12.045

8. Cruz AC and Gruber BL. Statins and osteoporosis:Can these lipid-lowering drugs alsobolster bones? Cleveland Clinic Journal of Medicine. 2002; 69:277-288. http://dx.doi. org/10.3949/ccjm.69.4.277

9. Liu $\mathrm{C}, \mathrm{Wu} \mathrm{Z}$ and Sun $\mathrm{H}$. The effect of simvastatin on mRNA expression of transforming growth factor- $\beta 1$, bone morphogenetic protein-2 and vascular endothelial growth factor in tooth extraction socket. International Journal of Oral Science. 2009; 1:90-98. http://dx.doi.org/10.4248/ijos.08011

10. Maciel-Oliveira N, Bradaschia-Correa V and Arana-Chavez VE. Early alveolar bone regeneration in rats after topicaladministration of simvastatin. Oral Surgery, Oral Medicine, Oral Pathology, Oral Radiology and Endodontology. 2011; 112:170-179. http://dx.doi. org/10.1016/j.tripleo.2010.08.027

11. WuZ, Liu C, Zang G and Sun H. The effect of simvastatin onremodelling of the alveolar bonefollowing tooth extraction. International Journal of Oral Maxillofacial Surgery. 2008; 37:170-176. http://dx.doi.org/10.1016/j. ijom.2007.06.018

12. Bosch C, Melsen B and Vargervik K. Importance of the critical-size bone defect in testing bone-generating materials. Journal of Craniofacial Surgery. 1998; 9:310-316. http:// dx.doi.org/10.1097/00001665-199807000-00004

13. Honma T, Itagaki T, Nakamura M, Kamakura S, Takahashi $\mathrm{I}$, Echigo $\mathrm{S}$ and Sasano Y. Bone formation in rat calvaria ceases within a limitedperiod regardless of completion of defect repair. Oral Diseases. 2008; 14:457-464. http://dx.doi. org/10.1111/j.1601-0825.2007.01401.x

14. Cieślik M, Mertas A, Morawska-Chochół A, Sabat D, Orlicki $\mathrm{R}$, Owczarek A et al. The evaluation of the possibilities of using PLGA co-polymer and its composites with carbon fibers or hydroxyapatite in the bone tissue regeneration process - in vitro and in vivo examinations. International Journal of Molecular Science. 2009; 10:3224-3234. http://dx.doi.org/10.3390/ ijms10073224

15. Udagawa A, Sato S, Hasuike A, Kishida M, Arai Y and Ito K. Micro-CT observation of angiogenesisin bone regeneration. Clinical and Oral Implant Research. 2012; 1-6. http://dx.doi. org/10.1111/j.1600-0501.2012.02458.x

16. Park J-B. The use of simvastatin in bone regeneration. Medicina Oral, Patología Oral y Cirugía Bucal. 2009; 14(9):485-488.

17. Grasser WA, Baumann AP, Petras SF, Harwood Junior HJ, Devalaraja R, Renkiewicz R et al. Regulation of osteoclast differentiation by statins. Journal of Musculoskeletal and Neuronal Interactions. 2003; 3:53-62. 